\title{
Evolución del consumo de antimicrobianos de uso restringido y tendencia de la susceptibilidad in vitro en el Hospital Regional de Concepción, Chile
}

\author{
Felipe E. Morales, Lorenzo A. Villa, Pola B. Fernández, Mariela A. López, Sergio Mella y Maritza Muñoz
}

\section{Universidad de Concepción, Concepción, Chile. Departamento de Farmacia} Farmacia Clínica (FEM, PBF, MAL,

HOPE Center, College of Pharmacy, University of Arizona, E.U.A. (LAV).

Hospital Clínico Regional de Concepción Dr. Guillermo Grant Benavente, Concepción, Chile. Unidad de Infectología (SM) Unidad de Farmacia (MM).

Establecimiento donde se realizó el estudio:

Hospital Clínico Regional de Concepción Dr. Guillermo Grant Benavente.

Declaración de conflictos de intereses: Sergio Mella Montecinos

declara recibir el pago de

honorarios por conferencias de

Merck Sharp \& Dohme (MSD) y el apoyo para viajes por parte de Astra Zeneca y MSD.

El resto de los autores declara no presentar conflictos de intereses.

Financiamiento: Dirección de Investigación, Universidad de Concepción. Proyecto DIUC No 2009.074.042-1.0

Recibido: 21 de septiembre de 2011

Aceptado: 18 de julio de 2012

Correspondencia a: Felipe Eduardo Morales León felimora@udec.c

\section{Introducción}

$\mathrm{E}$ s un hecho que la utilización de antimicrobianos ha mejorado la calidad y expectativas de vidas de las personas alrededor del mundo, reduciendo la morbilidad y mortalidad causadas por diferentes enfermedades infecciosas ${ }^{1}$. Los antimicrobianos son los fármacos más utilizados en el mundo, representando en los países desarrollados $35 \%$ del gasto sanitario total ${ }^{2}$. No obstante, se estima que entre 10 y $50 \%$ de las prescripciones de estos fármacos son inadecuadas ${ }^{3}$. Esta situación trae consecuencias directas al paciente, a la sociedad y a los sistemas de salud resultando en la aparición de resistencia bacteriana, fallas en los tratamientos, desarrollo de efectos adversos e interacciones farmacológicas y aumentando los costos de la atención sanitaria ${ }^{3-5}$.

Aunque un elevado porcentaje del consumo de estos fármacos ocurre en la comunidad, es en los hospitales donde se genera la mayor selección y diseminación de bacterias resistentes y multi-resistentes, requiriéndose en estos casos el uso de tratamientos antimicrobianos complejos y de elevado costo ${ }^{4,6}$.

El desarrollo de resistencia microbiana constituye un fenómeno de carácter multifactorial. Entre las numerosas estrategias utilizadas para disminuir este fenómeno se encuentran el desarrollo de las nuevas políticas relacionadas con la restricción en el uso de los antimicrobianos y la reducción de su consumo innecesario, junto con la vigilancia epidemiológica de la resistencia bacteriana local ${ }^{4}$. En Chile, así como en otros países, existen escasos datos sobre el uso de estos fármacos. Por tanto, tener acceso a esta información constituye un punto necesario para desarrollar medidas regulatorias e implementar nuevos programas de intervención que permitan reducir en parte este problema.

A fines del año 2003 se desarrolló en el Hospital Guillermo Grant Benavente de Concepción, un programa para regular la dispensación de antimicrobianos de última generación (carbapenémicos, inhibidores de $\square$-lactamasas y glicopéptidos) como una medida para controlar la utilización y consumo de estos fármacos. Sin embargo, a la fecha no existe evaluación de la utilización de éstos, por lo que se realizó un estudio para cuantificar el consumo de estos medicamentos y su posible impacto sobre los patrones de susceptibilidad bacteriana local. 


\section{Métodos}

\section{Diseño de estudio}

Se realizó un estudio observacional retrospectivo para cuantificar y determinar el consumo de antimicrobianos de uso restringidos y su impacto sobre la variación de la susceptibilidad bacteriana in vitro durante los años 2004 al 2008, en el Hospital Guillermo Grant Benavente de Concepción, establecimiento asistencial de alta complejidad, para todas las edades, con una capacidad de atención de 909 camas.

De forma paralela, se estimó el costo total asociado al consumo de este tipo de antimicrobianos, con el fin de establecer desde otra perspectiva su real impacto.

\section{Consumo de antimicrobianos}

Desde la creación de la Unidad de Infecciones Intrahospitalarias en este establecimiento, se definió como prioridad la restricción y control del uso de antimicrobianos, especialmente aquellos considerados de última generación, reservados para el tratamiento de pacientes con patología infecciosa más compleja. Este programa fue implementado a partir del año 2004, donde la prescripción de cada uno de los fármacos sometido a control debe ser evaluada previamente por un médico infectólogo. Por otra parte, el farmacéutico se encarga de dispensar estos fármacos previo chequeo de los requisitos necesarios para esto, registrando la dispensación y consumo de estos fármacos.

Para la evaluación del consumo de antimicrobianos de uso restringido se utilizó el sistema de clasificación propuesta por Collaborating Center for Drug Statistics Methodology (ATC/DDD), el que fue desarrollado por la Organización Mundial de la Salud (OMS) para comparar el uso de fármacos entre diferentes regiones del mundo, así como también, entre distintos establecimientos asistenciales de diversa complejidad ${ }^{7}$.

La dosis diaria definida por cada 100 días cama (DDD/100 días-cama) es el indicador de consumo de antimicrobianos más empleado en los hospitales ${ }^{7-9}$. Este parámetro permite cuantificar el consumo de estos fármacos en una población hospitalaria haciendo posible, entre otras cosas, estimar la presión ecológica ejercida por los antimicrobianos sobre la aparición y posterior propagación de bacterias resistentes, como un factor que contribuye de manera significativa sobre este fenómeno.

Los datos de consumo y el índice ocupacional, expresado como 100 días-cama, fueron obtenidos desde los registros de la Unidad de Farmacia y de Estadística del hospital.

Por otro lado, para evaluar la evolución de la susceptibilidad microbiana in vitro, se seleccionaron aquellas bacterias que resultan de interés clínico, debido a la frecuencia con que son aisladas desde pacientes hospitalizados, a su relación con infecciones intrahospitalarias y a las recomendaciones establecidas por el laboratorio biomédico del Instituto de Salud Pública del país ${ }^{10}$.

Los estudios de susceptibilidad in vitro fueron efectuados mediante la técnica de difusión en disco y microdilución en caldo (Vitek, BioMérieux o Phoenix, Becton-Dickinson). Todos los puntos de cortes utilizados durante los cuatro años de estudios fueron interpretados según lo establecido por el Clinical and Laboratory Standards Institute (CLSI) ${ }^{11}$. El informe semestral sobre susceptibilidad microbiana fue realizado por el laboratorio de microbiología del hospital.

De acuerdo con estos criterios mencionados, los microorganismos y antimicrobianos evaluados fueron: Staphylococcus aureus a cloxacilina, Enterococcus spp (Enterococcus faecium y Enterococcus faecalis), a ampicilina y vancomicina, Enterobacteriaceae (Proteus mirabilis, Klebsiella pneumoniae, Enterobacter cloacae, Escherichia coli) y Pseudomonas aeruginosa a cefepima, ciprofloxacina e imipenem, Acinetobacter baumannii a ampicilina/sulbactam, cefoperazona/sulbactam, ciprofloxacina e imipenem.

\section{Costo del consumo de antimicrobianos}

El costo del consumo de cada antimicrobiano estudiado se determinó utilizando el valor neto de cada unidad considerando la conversión del costo por cada DDD. Éste fue expresado en términos de dólar americano utilizando para ello el valor establecido por el Banco Central de Chile disponible a diciembre de 2008 (1 peso $=$ USD 0,00159 dólar).

\section{Análisis estadístico}

Se utilizó la $t$ de student para determinar las diferencias existentes entre el consumo anual de cada antimicrobiano respecto a lo utilizado durante el año 2004, el cual fue empleado como año de referencia. Para analizar el impacto del consumo de antimicrobianos sobre la susceptibilidad in vitro se consideró el consumo de estos fármacos, expresados en términos de $\mathrm{DDD} / 100$ días-cama y la susceptibilidad expresada como el porcentaje de cepas sensibles. Se estableció, en una primera instancia, la existencia de una relación simple entre ambos parámetros. Posteriormente, se calculó el coeficiente de correlación de Spearman, considerando sólo aquellas relaciones que presentaran un valor del coeficiente rho de Spearman igual o superior a 0,7 . Se consideraron aquellos resultados estadísticamente significativos con un valor de $\mathrm{p}<0,05$. Los análisis estadísticos fueron realizados con el software STATA versión 11.0 (StataCorp LD USA).

\section{Resultados}

La evolución del consumo de antimicrobianos de uso restringido experimentó un incremento significativo du- 
Tabla 1. Consumo y diferencia porcentual de los diferentes antimicrobianos de uso restringido durante el período 2004-2008

\begin{tabular}{|c|c|c|c|c|c|c|c|}
\hline \multirow[b]{2}{*}{ Antimicrobianos } & \multicolumn{5}{|c|}{ Consumo DDD/100 días-cama } & \multicolumn{2}{|c|}{ Variación 2004-2008 } \\
\hline & 2004 & 2005 & 2006 & 2007 & 2008 & $\%$ & $p$ \\
\hline Imipenem & 165,9 & 258,2 & 154,6 & 266,5 & 212,5 & +28 & 0,329 \\
\hline Meropenem & 477,8 & 131,1 & 448,2 & 424,6 & 335 & -30 & 0,170 \\
\hline Ertapenem & - & 8,2 & 75,4 & 83,3 & 134,5 & $+1.540^{*}$ & $<0,001$ \\
\hline Cefoperazona/sulbactam & 193 & 158,6 & 199,6 & 304,1 & 315,8 & +64 & 0,089 \\
\hline Cefepima & 77,9 & 218,6 & 126,1 & 103,4 & 97,3 & +25 & 0,373 \\
\hline Ampicilina/sulbactam & 36,8 & 85,8 & 89,2 & 168,7 & 63,5 & +73 & 0,362 \\
\hline Piperacilina/tazobactam & 10,5 & 17,3 & 26,2 & 54 & 68 & +548 & 0,001 \\
\hline Ciprofloxacina & 84,2 & 261,7 & 239,7 & 303,3 & 169,5 & +101 & 0,023 \\
\hline Teicoplanina & - & 1,3 & 2,4 & 7,6 & 4,5 & $+246^{*}$ & 0,204 \\
\hline Vancomicina & 589,6 & 716,8 & 745,9 & 847,2 & 740,1 & +26 & 0,118 \\
\hline Linezolid & - & 10,76 & 0,63 & 28,96 & 18,88 & $+75^{*}$ & 0,319 \\
\hline
\end{tabular}

rante los años 2004-2008 (35\%; $\mathrm{p}=0,005)$. Los servicios en los que se observó el mayor consumo de antimicrobianos fueron la Unidad de Tratamiento Intermedio (UTI) alcanzando $53,1 \%$, seguido de la Unidad de Cuidados Intensivos (UCI) con 32,4\%. Por otro lado, los servicios de Cirugía y Medicina fueron los que presentaron el menor consumo de antimicrobianos del hospital con 8,8 y $5,7 \%$ respectivamente.

Por su parte, los antimicrobianos de uso restringido que más incrementaron su consumo durante los cinco

Tabla 2. Costo anual del consumo de cada antimicrobiano calculado en términos de DDD/100 días-cama

\begin{tabular}{|lrrrrr}
\multicolumn{6}{l}{ Costo total anual del consumo de cada antimicrobiano (Dolar americano*) } \\
& $\mathbf{2 0 0 4}$ & $\mathbf{2 0 0 5}$ & $\mathbf{2 0 0 6}$ & \multicolumn{1}{c}{$\mathbf{2 0 0 7}$} & \multicolumn{1}{c}{$\mathbf{2 0 0 8}$} \\
Imipenem & $10.617,6$ & $16.524,8$ & $9.894,4$ & $17.056,0$ & $13.600,0$ \\
Meropenem & $25.801,2$ & $7.079,4$ & $24.202,8$ & $22.928,4$ & $18.090,0$ \\
Ertapenem & - & 549,4 & $5.051,8$ & $5.581,1$ & $9.011,5$ \\
Cefoperazona/sulbactam & $7.527,0$ & $6.185,4$ & $7.784,4$ & $11.859,9$ & $12.316,2$ \\
Cefepima & $2.492,8$ & $6.995,2$ & $4.035,2$ & $3.308,8$ & $3.113,6$ \\
Ampicilina/sulbactam & 552,0 & $1.287,0$ & $1.338,0$ & 2530,5 & 952,5 \\
Piperacilina/tazobactam & 934,5 & $1.539,7$ & $2.331,8$ & 4806,0 & $6.052,0$ \\
Ciprofloxacina & 252,6 & 785,1 & 719,1 & 909,9 & 508,2 \\
Teicoplanina & - & 39,0 & 72,0 & 228,0 & - \\
Vancomicina & $4.893,7$ & $5.949,4$ & $6.191,0$ & $7.031,7$ & $6.142,8$ \\
Linezolid & - & $1.678,6$ & 98,3 & $4.517,8$ & $2.945,2$ \\
Total & $53.073,4$ & $48.613,0$ & $61.718,8$ & $80.758,1$ & $72.867,0$
\end{tabular}

*1 peso $=$ USD 0,00159 dólar. Fuente Banco Central de Chile. Diciembre de 2008. años, fueron los glicopéptidos con $37 \%$ del consumo total, seguidos por los carbapenémicos los cuales representaron $30,3 \%$

Tomando en cuenta los antimicrobianos estudiados, se observó un elevado consumo de cefalosporinas de tercera generación y penicilinas asociadas a inhibidores de $\square$-lactamasas. Destacó además, el incrementó en el uso de aquellos antimicrobianos considerados como de última generación o reservados para situaciones más complejas, siendo ertapenem, piperacilina/tazobactam y ciprofloxacina aquellos que experimentaron un aumento de consumo más significativo (Tabla 1).

\section{Gasto en antimicrobianos}

Varió ampliamente a lo largo de los cinco años estudiados. Sin embargo, fue durante el año 2007 donde se generó el mayor gasto relacionado con la utilización de estos fármacos, destacando principalmente imipenem y linezolid (Tabla 2).

\section{Variación de la susceptibilidad in vitro}

No se observó una variación estadísticamente significativa de susceptibilidad en las cepas de microorganismos grampositivos estudiados. Sí se observó una baja susceptibilidad a cloxacilina en cepas de Staphylococcus spp, aun cuando, es importante señalar que en los reportes de susceptibilidad microbiana entregados por el laboratorio del hospital se incluyen muestras provenientes de pacientes hospitalizados, sin diferenciar si corresponden a muestras de infecciones intrahospitalarias o de origen comunitario. Respecto a la susceptibilidad in vitro observada para cepas de Enterococcus spp a ampicilina, se observó diferencia entre E. faecalis y E. faecium, ya que para la primera 
especie hubo $92 \%$ de cepas susceptibles, mientras que para la segunda, todas las cepas fueron resistentes a este antimicrobiano. Por otro lado, frente a vancomicina se observó un disminución en la susceptibilidad de las cepas de E. faecium a lo largo de los años, alcanzando sólo 67\% en el año 2008, a diferencia de las cepas de E. faecalis que mantuvieron una alta susceptibilidad observándose $98 \%$ en el mismo año (Tabla 3 ).

Respecto a la susceptibilidad in vitro de las Enterobacteriaceae, cepas de Escherichia coli aisladas de fluidos distintos a la orina mostraron una clara tendencia a la disminución de la susceptibilidad a ceftriaxona, desde $87 \%$ en 2004 , a $40 \%$ en el 2008 . Por su parte, en E. coli proveniente de muestras de orina, se observó una susceptibilidad sin variaciones estadísticamente significativas en el tiempo. En Klebsiella pneumoniae se observó una baja susceptibilidad in vitro a ceftriaxona, ampicilina/sulbactam y ciprofloxacina. Es importante tener en cuenta la baja susceptibilidad a cefepima observada durante el año 2008 en todas las bacterias gramnegativas, con excepción de $E$. coli aislada de muestras de orina (Tabla 4).

Respecto a los bacilos gramnegativos no fermentadores, se observaron variaciones importantes, especialmente para Pseudomonas aeruginosa, ya que durante el año 2008 se observó una baja susceptibilidad in vitro a cefepima e imipenem con 52 y $72 \%$ de cepas susceptibles, respectivamente. Se determinó además, un descenso significativo de la susceptibilidad de este microorganismo a imipenem, desde $91 \%$ durante el 2004 a 72\% durante el año 2008 $(\mathrm{p}=0,038)$. Respecto a la susceptibilidad in vitro de Acinetobacter baumannii, se observó una elevada susceptibilidad a las diferentes combinaciones de penicilinas y sulbactam, donde la combinación cefoperazona/sulbactam fue activa en $95 \%$ de las cepas, mientras que $88 \%$ de ellas fueron sensibles a ampicilina/sulbactam (Tabla 4).

\section{Discusión}

El presente estudio aporta nuevos datos locales relacionados con el aumento del consumo de antimicrobianos y la variación de la susceptibilidad bacteriana in vitro.

Se determinó que entre los años 2004 y 2008 existió un aumento considerable y sostenido del consumo de antimicrobianos clasificados como de uso restringido en este hospital. Entre las probables causas de este aumento se podría señalar el incremento en la tasa ocupacional observada durante el período de estudio, el aumento en la capacidad, entendido como el incremento en el número de camas de cuidados intensivos (12 nuevas camas en la Unidad Coronaria en el año 2005 y 10 en la UCI en el año 2008) y, probablemente, a la disminución en las horas de trabajo del especialista infectólogo, pudiendo esto llevar a una disminución en la capacidad de control del programa de prescripción de estos antimicrobianos y por tanto, a un aumento significativo en su consumo.

Estos hallazgos son similares a los encontrados en estudios europeos, donde se observó un incremento en el consumo global de estos fármacos. Al respecto, en Italia, se estudió durante tres años el consumo de antimicrobianos en un hospital público, determinándose un aumento de $18 \%$ en el consumo de estos fármacos ${ }^{15}$. Por su parte,

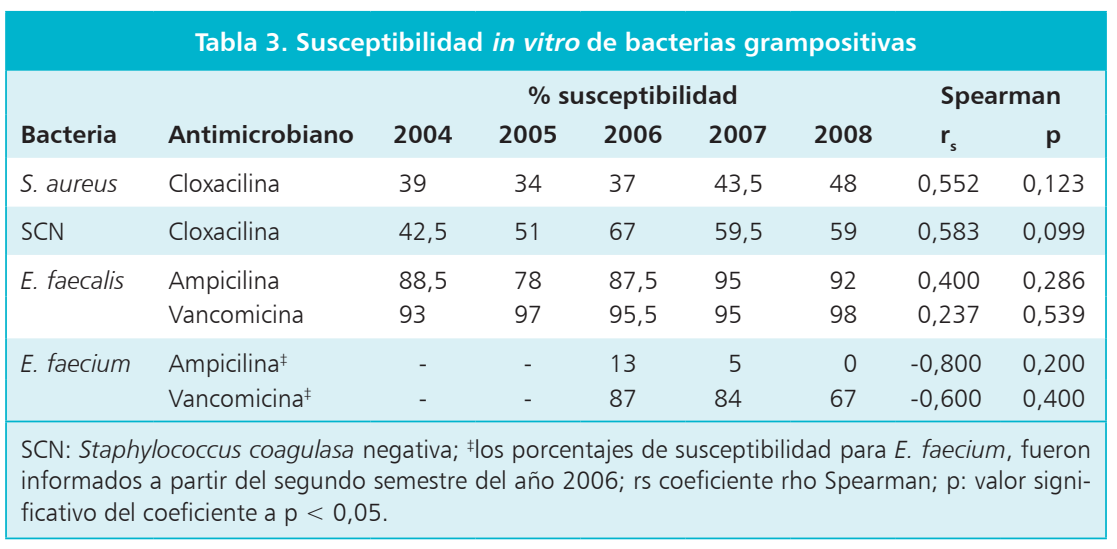

\begin{tabular}{|c|c|c|c|c|c|c|c|c|}
\hline \multirow[b]{2}{*}{ Bacteria } & \multirow[b]{2}{*}{ Antimicrobiano } & \multicolumn{5}{|c|}{$\%$ susceptibilidad } & \multicolumn{2}{|c|}{ Spearman } \\
\hline & & 2004 & 2005 & 2006 & 2007 & 2008 & rs & $p$ \\
\hline \multirow[t]{2}{*}{ P. mirabillis } & Cefepima & 80 & 89 & 91 & 73 & 53 & $-0,550$ & 0,125 \\
\hline & Ampicilina/sulbactam & 40 & 46 & 79 & - & - & 0,943 & 0,005 \\
\hline \multirow[t]{4}{*}{ K. pneumoniae } & Ceftriaxona & 39 & 44 & 24 & 31 & 21 & $-0,778$ & 0,014 \\
\hline & Cefepima & 46 & 66 & 47 & 46 & 22 & $-0,770$ & 0,015 \\
\hline & Cefoperazona/sulbactam & 54 & 60 & 53 & 45 & 48 & $-0,633$ & 0,067 \\
\hline & Ciprofloxacina & 68 & 72 & 43 & 45 & 47 & $-0,745$ & 0,021 \\
\hline \multirow[t]{3}{*}{ E. cloacae } & Cefoperazona/sulbactam & 60 & 71 & 60 & 68 & 83 & 0,763 & 0,017 \\
\hline & Ciprofloxacina & 61 & 61 & 62 & 74 & 71 & 0,826 & 0,006 \\
\hline & Imipenem & 99 & 100 & 99 & 100 & 100 & 0,596 & 0,090 \\
\hline \multirow[t]{5}{*}{ E. coli ${ }^{\dagger}$} & Ceftriaxona & 87 & 82 & 81 & 54 & 40 & $-0,800$ & 0,010 \\
\hline & Amikacina & 88 & 94 & 97 & 95 & 76 & 0,109 & 0,781 \\
\hline & Cefepima & 94 & 87 & 86 & 80 & 67 & $-0,783$ & 0,013 \\
\hline & Ciprofloxacina & 82 & 76 & 100 & 99 & 65 & $-0,549$ & 0,126 \\
\hline & Imipenem & 99 & 100 & 100 & 99 & 100 & 0,559 & 0,118 \\
\hline \multirow[t]{5}{*}{ E. coli ${ }^{\ddagger}$} & Ceftriaxona & 89 & 89 & 90 & 78 & 100 & $-0,167$ & 0,668 \\
\hline & Amikacina & 88 & 96 & 97 & 93 & 100 & 0,434 & 0,243 \\
\hline & Cefepima & 89 & 89 & 97 & - & 84 & 0,09 & 0,848 \\
\hline & Ciprofloxacina & 79 & 82 & 80 & 78 & 76 & $-0,150$ & 0,700 \\
\hline & Imipenem & 99 & 98 & 100 & 99 & 99 & 0,158 & 0,685 \\
\hline \multirow[t]{2}{*}{$P$. aeruginosa } & Cefepima & 88 & 76 & 87 & 72 & 52 & $-0,633$ & 0,067 \\
\hline & Imipenem & 91 & 93 & 87 & 76 & 72 & $-0,695$ & 0,038 \\
\hline \multirow[t]{3}{*}{ A. baumannii } & Ampicilina/sulbactam & 60 & 56 & 45 & 78 & 88 & 0,683 & 0,042 \\
\hline & Ciprofloxacina & 40 & 39 & 35 & 19 & 15 & $-0,720$ & 0,029 \\
\hline & Cefoperazona/sulbactam & 73 & 73 & 80 & 82 & 95 & 0,717 & 0,030 \\
\hline
\end{tabular}


Müller-Pebody ${ }^{13}$ y Jensen ${ }^{3}$ establecieron un incremento en el consumo de antimicrobianos en distintos hospitales daneses, observando un aumento del consumo de $18 \%$ entre los años 1997-2001 y de 43\% entre los años 2001-2007.

En nuestro hospital se encontró un aumento en el consumo en todas las áreas clínicas estudiadas, siendo éste mayor en la UTI y UCI. Al respecto, el elevado nivel del consumo de antimicrobianos en estos servicios clínicos, responde necesariamente al tipo de pacientes, ya que más de $70 \%$ de ellos presentan una o más infecciones que a menudo son causadas por microorganismos con grado variable de resistencia ${ }^{13,14}$.

Los antimicrobianos más consumidos fueron los glicopéptidos y carbapenémicos.

El alto consumo observado para glicopéptidos se relaciona con un elevado número de prescripciones necesarias para el tratamientos de infecciones producidas por cepas de Staphylococcus aureus resistente a meticilina (SARM), en un hospital con una elevada endemia de este microorganismo. Algunos autores destacan que más de $50 \%$ de las prescripciones de vancomicina son utilizadas como tratamiento empírico o profiláctico, siendo esta indicación generalmente inapropiada ${ }^{12,15}$. Por otro lado, la aparición de cepas de enterococos resistentes a glicopéptidos determinó el aumento en el consumo de linezolid a partir del año 2005.

Por su parte, el incremento en el consumo de carbapenémicos durante el período estudiado se debió a un aumento en las infecciones causadas por bacilos gramnegativos productores de $\square$-lactamasas de espectro extendido (BLEE), donde imipenem, meropenem y más recientemente ertapenem, han demostrado tener una excelente actividad en el manejo de infecciones graves causadas por estas especies ${ }^{16}$. Se observó un bajo consumo de ertapenem durante el período de estudio, pese a ser éste el antimicrobianos que experimentara el mayor incremento del consumo en el mundo, debido a su favorable perfil farmacocinético y a la capacidad de ser empleado en antibioterapias ambulatorias ${ }^{17}$.

Las quinolonas representan $11 \%$ del consumo total acumulado de los antimicrobianos de uso restringidos durante los cinco años estudiados. Al respecto, ciprofloxacina ha experimentado un aumento significativo de su consumo entre los años 2004 y 2008, debido al amplio espectro de acción y su excelente actividad bactericida, principalmente en el tratamiento de infecciones no bacteriémicas producidas por microorganismos productoras de $\mathrm{BLEE}^{16}$. Aunque algunos investigadores sostienen que un aumento en el consumo de ciprofloxacina tiene un impacto directo en el desarrollo de resistencia bacteriana, particularmente en el desarrollo de cepas de E. coli resistentes a quinolonas y cefalosporinas, en nuestro hospital no fue posible observar estas relaciones ${ }^{17,18}$.

El consumo de penicilinas asociados a un inhibidor de -lactamasas, tales como piperacilina/tazobactam y ampicilina/sulbactam (2004-2007) también experimentó un incremento en su consumo, debido probablemente a la actividad que presenta sulbactam sobre cepas de $A$. baumannii ${ }^{19}$.

Numerosos autores reconocen una asociación significativa entre el aumento del consumo de cefalosporinas de amplio espectro y una mayor incidencia de infecciones nosocomiales causadas por cepas de $K$. pneumoniae productoras de $\mathrm{BLEE}^{20}$. Es importante tener en cuenta este punto, debido a la disminución de la susceptibilidad a cefepima observada en el período de estudio, principalmente durante el año 2008, en cepas de E. coli (muestras diferentes a orina), $P$. aeruginosa y K. pneumoniae. Según Hsueh $^{21}$, desde la introducción de cefepima en un hospital universitario en Taiwan durante el año 1999, existió un aumento de la resistencia a este antimicrobiano en cepas de $P$. aeruginosa, $K$. pneumoniae y $A$. baumannii. Por su parte, Mella ${ }^{22}$, analizó durante el año 2001, numerosas cepas de $K$. pneumoniae y E. coli provenientes de muestras aisladas de hospitales chilenos, encontrando que sólo 2,6\% de los aislados de K. pneumoniae y $26 \%$ de $E$. coli fueron resistentes a cefepima. En ese momento, los autores señalaron que cefepima constituía una excelente alternativa para el tratamiento de infecciones causadas por bacilos gramnegativos resistentes a cefalosporinas de tercera generación. Más recientemente, se ha identificado una mayor tasa de resistencia en cepas de E. coli y $K$. pneumoniae a cefepima. Al respecto, la producción de $\square$-lactamasas de amplio espectro confiere resistencia a la mayoría de las cefalosporinas, incluyendo aquellas de tercera y cuarta generación. La presencia de estas enzimas, podría explicar la baja susceptibilidad observada a cefepima, ceftriaxona y, en menor medida, a derivados de penicilina asociados a inhibidores de - -lactamasas y a fluoroquinolonas. Además, esto podría estar contribuyendo al aumento en el consumo de carbapenémicos ya que se considera a estos fármacos como la terapia de primera línea contra infecciones causadas por bacterias productoras de $\square$-lactamasas de amplio espectro ${ }^{20,23}$.

Por su parte, $P$. aeruginosa corresponde a un patógeno comúnmente aislado en infecciones nosocomiales, mostrando en este estudio una baja susceptibilidad a cefepima (52\% de las cepas susceptibles durante el año 2008) e imipenem con una disminución significativa de la susceptibilidad desde $91 \%$ durante el año 2004 hasta 72\% en el $2008(p=0,038)$. Estos antecedentes son consistentes con los hallazgos descritos por otros autores ${ }^{24,25}$.

Las medidas tendientes a mitigar el impacto del uso de antimicrobianos deben incluir la implementación de programas de vigilancia asociados a métodos efectivos de medición de la susceptibilidad bacteriana, de manera que permitan conducir a terapias empíricas efectivas. Un concepto novedoso, que pudiera considerarse válido, es crear 
un programa para la rotación cíclica de antimicrobianos, el que debe ser implementado como una estrategia adicional al cumplimiento estricto de las medidas de prevención y control de infecciones intrahospitalarias, mitigando en parte el rápido desarrollo de resistencia microbiana ${ }^{26}$.

\section{Limitaciones}

La DDD es una unidad técnica de medida para comparar el consumo de fármacos en diferentes instituciones y en distintos períodos de tiempo, siendo su valor independiente de los cambios en el precio y de la forma farmacéutica. Corresponde a un indicador de los cambios en los hábitos terapéuticos, pero no es capaz de identificar las causas específicas de estos cambios. En el cálculo de DDD se utilizaron datos provenientes de la dispensación, por lo que refleja el consumo y el grado de prescripción de estos fármacos. Por tanto, la DDD puede ser modificada por variaciones en la frecuencia de las enfermedades y en los hábitos de prescripción por parte de los médicos.

En este estudio se muestra un aumento en el consumo de ciertos agentes antimicrobianos en relación con un cambio significativo en el patrón de susceptibilidad. Aunque existe una correlación entre estas dos variables, la relación no es necesariamente causal. No es posible predecir lo que sucederá con la susceptibilidad microbiana in vitro respecto a los cambios en el consumo de estos antimicrobianos ya que este no es el único factor involucrado.

\section{Conclusiones}

El aumento en el uso de antimicrobianos de uso restringidos podría estar influenciada por numerosos factores tales como el aumento de la capacidad de hospitalización, especialmente en las unidades que atienden a pacientes críticos, la falta de especialistas que permitan un mejor control en la utilización de estos medicamentos, un aumento de las enfermedades endémicas producidas por cepas de SARM y la fuerza representada por el uso de cefalosporinas de tercera generación para el tratamiento de diversas infecciones (neumonía, meningitis, peritonitis, e infecciones del tracto urinario), que ligado a una elevada presión selectiva, contribuye a la aparición de bacilos gramnegativos multi-resistentes, principalmente productores de BLEE o de Amp-C.

El comportamiento de los microorganismos a diferentes antimicrobianos es complejo, por lo que es necesario mantener una vigilancia adecuada y un correcto registro de la información, que permita un adecuado análisis epidemiológico para promover, a través del diseño de una política local y quizás nacional, el uso racional de estos medicamentos.

\section{Resumen}

En Chile no existen estudios para cuantificar el consumo de antimicrobianos de uso restringido al interior de los hospitales. Objetivo: Analizar el consumo de antimicrobianos de uso restringido en pacientes hospitalizados durante los años 2004-2008 en el Hospital Guillermo Grant Benavente de Concepción. Además, se analizaron las correlaciones entre este consumo y el patrón de susceptibilidad in vitro. Material y Método: Se diseñó un estudio observacional retrospectivo empleando las DDD/100-días-cama para evaluar el consumo de antimicrobianos, y el informe acumulado de susceptibilidad in vitro entregado por el laboratorio local, para analizar la evolución de la susceptibilidad. Resultados: El consumo de antimicrobianos se incrementó en $35 \%(\mathrm{p}=0,005)$ durante los años 2004-2008, donde los más consumidos fueron glicopéptidos (37\%) y carbapenémicos (30\%). Estos resultados se pueden explicar por la aparición de cepas de Staphylococcus aureus resistente a meticilina y bacilos gramnegativos productores de $\square$-lactamasas de espectro extendido. Además, se observó una disminución de la susceptibilidad de Pseudomonas aeruginosa a imipenem $(\mathrm{p}=0,038)$ y de Klebsiella pneumoniae a ciprofloxacina $(\mathrm{p}=0,021)$. Conclusiones: El consumo total de antimicrobianos de uso restringido se incrementó significativamente en los servicios clínicos más complejos, observándose una disminución de la susceptibilidad de algunos bacilos gramnegativos. El monitoreo de la prescripción de antimicrobianos así como de la susceptibilidad in vitro local constituyen medidas esenciales para promover el uso racional de antimicrobianos.

\section{Referencias bibliográficas}

1.- Livermore D M. Bacterial resistance: Origins, epidemiology, and impact. Clin Infect Dis 2003; 36: S11-S23.

2.- Valenzuela M T, Prat M S, Santolaya M E, Sakurada A, García P, González P, et al. Implementación de una red nacional para la vigilancia de resistencia de agentes patógenos a antimicrobianos según síndromes clínicos.
[Starting a national surveillance network of antibiotic resistance classified by clinical syndromes]. Rev Chilena Infectol 2003; 20: 119-25.

3.- Jensen U S, Skjot-Rasmussen L, Olsen S S, Frimodt-Moller N, Hammerum A M, DANRES Study Group. Consequences of increased antibacterial consumption and change in pattern of antibacterial use in Danish hospitals. J Antimicrob Chemother 2009; 63 (4): 812-5.
4.- Neu H C. The crisis of antibiotic resistance. Science 1992; 257 (5073): 1064-73.

5.- Mc Gowan J E. Economic impact of antimicrobial resistance. Emerg Infect Dis 2001; 7 (2): 286-92.

6.- Tenover F C. Mechanisms of antimicrobial resistance in bacteria. Am J Med 2006; 119 (6, Supplement 1): S3-S10.

7.- Kuster S, Ruef C, Laderberger B, Hintermann A, Deplazes C, Neuber L, et al. 
Quantitative antibiotic use in hospitals: Comparison of measurements. Literature review and recommendations for a standard of reporting. Infection 2008; 36: 549-59.

8.- WHO. WHO Collaborating center for drug statistic methodology ATC/DDD index. http:// www.whocc.no/atc_ddd_index/[accedido 17 mayo de 2011].

9.- Roger L W. Chapter 6: How do measurements of antibiotic consumption. Gould I, Meer Vd. Antibiotic Policies.Theory and Practice, 1 st ed. New York: Springer; 2005, p.75-100.

10.- ISP. Prueba de Susceptibilidad Antimicrobiana por Difusión en Agar. Santiago de Chile: Instituto de Salud Pública ISP - Chile, 2004.

11.- CLSI. Performance Standards for Antimicrobial Susceptibility Testing, M100-S15. Wayne PA: Clinical and Laboratory Standards Institute 2005.

12.- Vaccheri A, Silvani MC, Bersaglia L, Motola D, Strahinja P, Vargiu A, et al. A 3 year survey on the use of antibacterial agents in five Italian hospitals. J Antimicrob Chemother 2008; 61 (4): 953-8.

13.- Müller-Pebody B, Muscat M, Pelle B, Klein B M, Brandt C T, Monnet D L. Increase and change in pattern of hospital antimicrobial use, Denmark, 1997-2001. J Antimicrob Chemother 2004; 54 (6): 1122-6.

14.- Siddiqui S, Hussein K, Manasia R, Samad A, Salahuddin N, Zafar A, et al. Impact of antibiotic restriction on broad spectrum antibiotic usage in the ICU of a developing country. J Pak Med Assoc 2007; 57 (10):
484-7.

15.- Kaki R, Elligsen M, Walker S, Simor A, Palmay L, Daneman N. Impact of antimicrobial stewardship in critical care: a systematic review. J Antimicrob Chemother 2011; 66 (6): 1223-30

16.- Tumbarello M, Sanguinetti M, Montuori E, Trecarichi EM, Posteraro B, Fiori B, et al. Predictors of mortality in patients with bloodstream infections caused by extended-spectrum- $\beta$-lactamase-producing Enterobacteriaceae: importance of inadequate initial antimicrobial treatment. Antimicrob Agents Chemother 2007; 51 (6): 1987-94.

17.- Zhanel G G, Wiebe R, Dilay L, Thomson K, Rubinstein E, Hoban D J, et al. Comparative review of the carbapenems. Drugs 2007; 67 (7): 1027-52.

18.- Bermejo J, Bencomo B, Armesi N, Lesnaberes P, Borda N, Notario R. Alta correlación entre el consumo de ciprofloxacina y la prevalencia de Klebsiella pneumoniae productora de $\beta$-lactamasas de espectro extendido. Rev Chilena Infectol 2006; 23: 316-20.

19.- Diomedi A. Infecciones por Acinetobacter baumannii pan-resistente. Consideraciones epidemiológicas y de manejo antimicrobiano actualizado. Rev Chilena Infectol 2005; 22 : 298-320.

20.- Martínez J A, Aguilar J, Almela M, Marco F, Soriano A, López F, et al. Prior use of carbapenems may be a significant risk factor for extended-spectrum $\beta$-lactamase-producing
Escherichia coli or Klebsiella spp. in patients with bacteraemia. J Antimicrob Chemother 2006; 58 (5): 1082-5.

21.- Hsueh P, Chen W, Luh K. Relationships between antimicrobial use and antimicrobial resistance in Gram-negative bacteria causing nosocomial infections from 1991-2003 at a university hospital in Taiwan. Int $\mathrm{J}$ Antimicrob Agents 2005; 26 (6): 463-72.

22.- Mella S, Zemelman C, Bello H, Domínguez M, González G, Zemelman R. Propiedades microbiológicas, clasificación y relación estructura-actividad de cefalosporinas e importancia de las cefalosporinas de cuarta generación. Rev Chilena Infectol 2001; 18: 7-19.

23.- Lai C, Wang Y, Chu C, Tan K, Lu L, Lee C, et al. Correlation between antibiotic consumption and resistance of Gram-negative bacteria causing healthcare-associated infections at a university hospital in Taiwan from 2000 to 2009. J Antimicrob Chemother 2011; 66 (6): 1374-82

24.- Van Eldere J. Multicentre surveillance of Pseudomonas aeruginosa susceptibility patterns in nosocomial infections. J Antimicrob Chemother 2003; 51 (2): 347-52.

25.- Bouza E, García-Garrote F, Cercenado E, Marín M, Díaz S. Pseudomonas aeruginosa: a survey of resistance in 136 hospitals in Spain. Antimicrob Agents Chemother 1999; 43 (4): 981-31.

26.- Gyssens I C. Antibiotic policy. Int J Antimicrob Agents 2011; 38S: 11-20. Review. 